\title{
The Importance of Effective Regulatory Control in the Pharmaceutical Industry in China
}

\author{
Qianyin Liu ${ }^{1, *}$ Haozhe $\mathrm{Zhu}^{1}$
}

\author{
${ }^{1}$ University College London, London, England, WC1E $6 B T$ \\ ${ }^{1}$ YK Pao School, Shanghai, China, 201620 \\ *Corresponding author. Email: sop.saso@ucl.ac.uk
}

\begin{abstract}
Given the continuous expansion and development of the drug market caused by the increasing demand for drugs, the socialized large scale production of the drug industry has changed from the leading industry monopoly to a fierce competition between large, medium and small-sized enterprises. Due to the sharp increase in the scope of regulatory objects, the quality and safety of each drug which will be circulated on the market should attract more attention from more relevant people in the pharmaceutical industry. At present, China's pharmaceutical industry administrative departments include the State Food and Drug Administration, the National Family Planning and Health Commission, and the National Ministry of Human Resources and Social Security. Among them, the Food and Drug Administration, as the competent industry department, and the drug supervision and administration departments of the people's governments across the country are responsible for the drug supervision and administration work within their respective administrative areas. This paper discusses the contents of the Chinese pharmaceutical management system, the significance of the pharmaceutical industry supervision, and basing on the national conditions and author experience, giving strategies and suggestions on how to improve the effective regulatory control of the pharmaceutical industry[9].
\end{abstract}

Keywords: drug regulation, drug regulation, drug safety

\section{INTRODUCTION}

The pharmaceutical industry develops and manufactures medicines or pharmaceutical drugs for usage as medications to be delivered to patients in order to cure, vaccinate, or relieve symptoms. Consider its important role played in society concerning human life, effective regulatory controls must be implemented to provide safeguards for patients while treating the diseases.

The United States Food and Drug Administration (FDA), established in 1906, and many other drug administration authorities around the world are responsible for the job of approving new drugs, control the sale of current products and recall drug products on the market with regard to concerns about product quality, safety, or efficacy.

As Paracelsus has stated, "all things are poison, and nothing is without poison; only the dose makes a thing not a poison". That suggests that the dose and concentration of the active pharmaceutical ingredient (API) must be carefully controlled. If the amount of API in the drug taken does not meet the minimum effective concentration, no therapeutic effect would occur; if overdoses of medicine are taken, it becomes a poison. Therefore, it is administered by the Food and Drug Administration that drug manufacturers have to clearly label the dosage and adverse effects that might occur on the written brochure of prescribing information for physicians and patients [1]. Not only do the drugs have to be approved, but the Full Prescribing Information (FPI) would also have to be approved before launching on the market in order to protect patients from disorders caused by the misuse of drugs [2].

Besides the regulation on the prescribing information, the regulations from the Food and Drug Administration authorities range widely from discovery to approval and even the recall of the drug. 
This paper focuses on the importance of effective regulations in the pharmaceutical industry. In order to do that, regulations issued by the Food and Drug Administration in use nowadays would be introduced first. Then, the effectiveness of these regulations would be examined. In addition to these, real-life examples of successfully and unsuccessfully regulated medicines would be used to illustrate the effects of these regulatory controls valid these days. In other words, questions of "what regulations would take place before a medicine goes onto the market", "why are regulations necessary for the pharmaceutical industry", "what are the results of the regulatory controls", and "what are some areas that the law and regulations nowadays can be improved on".

\section{LITERATURE REVIEW}

\subsection{Drug Development Process}

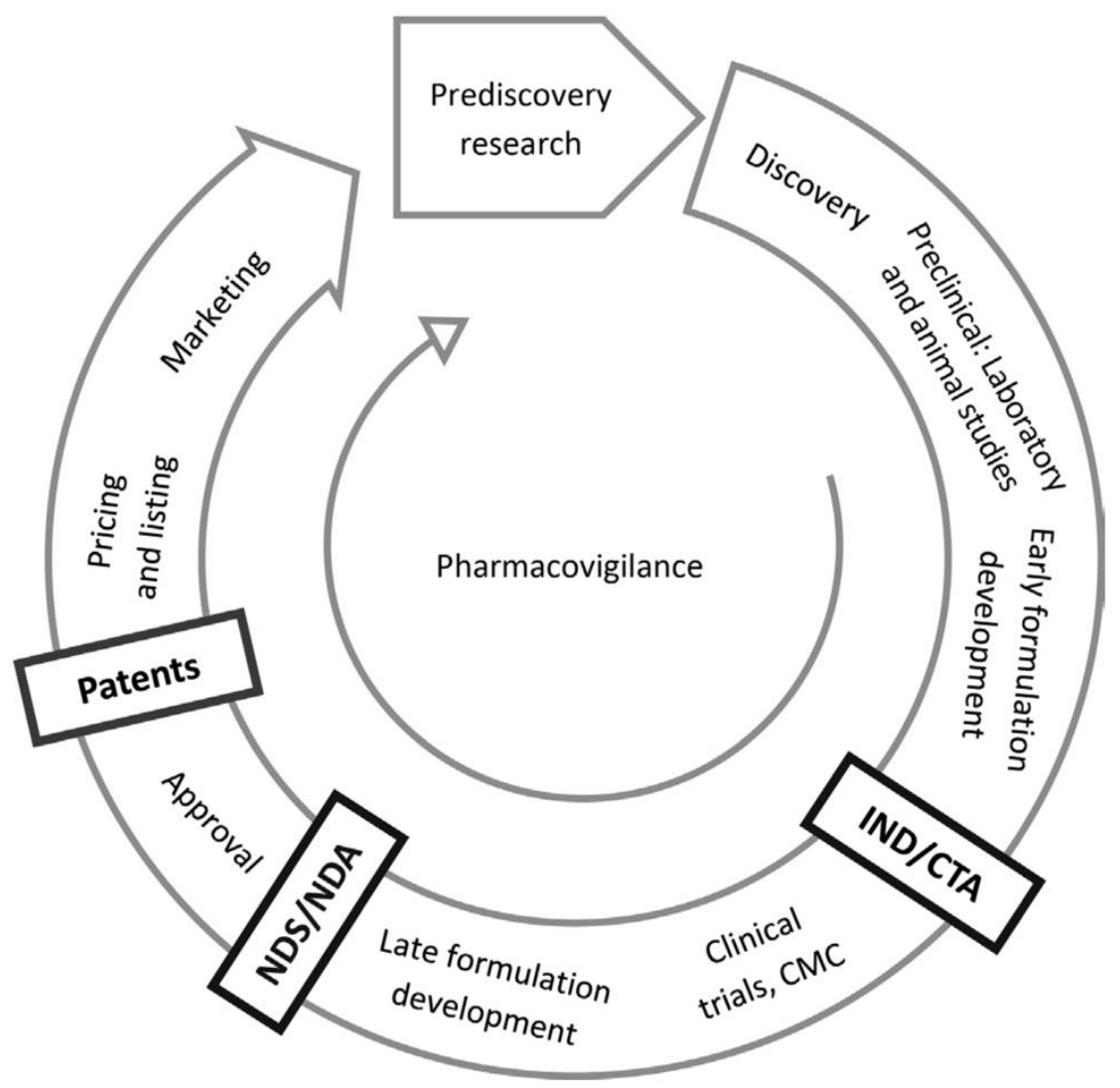

Figure 1 The Pharmaceutical Product Life Cycle Showing Intersection of the Regulator at Different Points: IND/CTA, NDS/NDA, and Patents [4]

If the FDA approves the IND, the drug company can start to conduct clinical trials. Usually, clinical trials are divided into three different phases. In Phase I, a small group of volunteers about 20 to 100 healthy volunteers recruited to test the safety issues of the new drug. In this phase, the safety of the drug is first examined to make sure no life risks will be aroused by this drug. Following, Phase II monitors the efficacy of the newly
Figure 1 shows the drug development process in a flowchart. For a drug to be successfully approved by the administration authorities, the first step is a long period of preclinical research to find out a drug suitable for treating a certain kind of disease from thousands of potential compounds. The scientists at the innovative drug companies would then need to identify a potential drug's defined mechanism of action regarding how the chemical works. As regulated by the Public Health Service Act (PHSA), Section 351, the manufacturer then sends an Investigational New Drug (IND) application to the FDA if the drug has got promising results from the previous studies. The IND application is a comprehensive document that comprises information on animal pharmacology and toxicity research, manufacturing and quality details, and clinical protocols [3]. 
range of potential drugs failed to be approved in these stages.

Only if all the results obtained support the approval of this new drug, the drug company would submit a New Drug Application (NDA) to the FDA. As soon as the approval is obtained, the manufacturer can begin to market the product[2]. Meanwhile, the FDA would continue to monitor the situation of the drug after its release and prepare to recall the medicine at any time.

\subsection{Failures of Drugs}

In the development of the pharmaceutical industry, there is a list of drugs that have unfortunately cause unpredicted consequences. Adverse effects of the drugs range from defects in birth, strokes, hepatitis, death and so on [5]. The existence of these drugs stresses the importance of effective regulatory control of the pharmaceutical industry again. With a more carefully designed regulatory system, some of the problems occur in the past could be avoided to reduce the number of losses.
In addition to these physiological consequences, the economic loss caused by the recall of the drug is also uncountable. Once the drug has been recalled, all of the investment, as much as a few billion, would evaporate. Plus, these unqualified medicines would also cause indelible consequences to patients on the social aspect. For instance, the life of children suffering phocomelia caused by thalidomide would be extremely difficult. Considering the wide range of fields influenced by a dosage of medicine, effective regulatory controls of the pharmaceutical industry are necessary.

\subsubsection{Phocomelia caused by Thalidomide}

Many children born in the 1960's suffered from a disease called phocomelia as a side effect of the medication called thalidomide that causes limb shortening or absence. Thalidomide started to prevail as a hypnotic pill when people around the world suffer from insomnia in the post-war era. It was advertised as "completely safe" for everyone, including mother and child, "even during pregnancy".

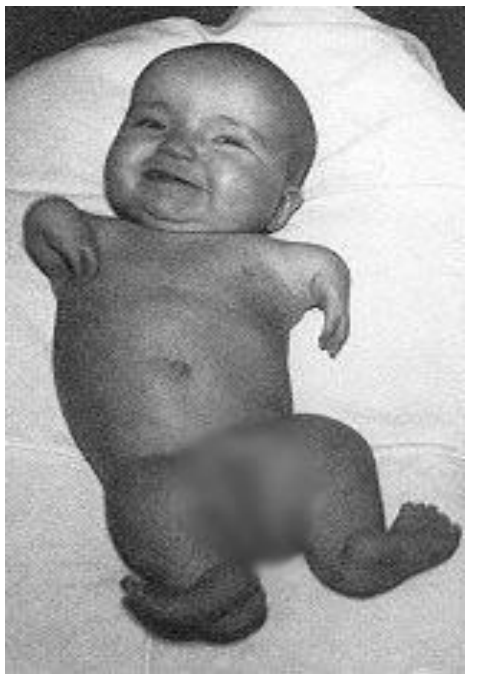

Figure 2 A newborn child with Phocomelia caused by Thalidomide [6]

Sadly, tragedy followed its release. After these pregnant women who have taken thalidomide delivered, hundreds of cases of babies with shortened, absent, or flipper-like limbs as shown in Figure 2 are reported. The drug's producers, who had ignored concerns of birth abnormalities were eventually forced to halt the distribution of thalidomide in Europe [5]. Following the global thalidomide crisis, governments all over the world, including the United States, revised their drug regulation processes and legislation. In 1962, the 1962 Drug Amendments are enacted, and clinical trials are forced to be taken place to serve as the scientific testing evidence for the FDA's approval of new drugs [7].

\subsubsection{Strokes caused by Phenylpropanolamine}

Phenylpropanolamine is known as a psychoactive drug. Although very prevalent since the early decades of the $20^{\text {th }}$ century, FDA had never formally approved it. That is, until a Yale University study in 2000 discovered a link between it and cardiac disorders and stroke, particularly in women. The FDA estimates that between 200 to 500 strokes per year would have been prevented if phenylpropanolamine was replaced. Lawsuits have been brought against a variety of medicines firms, and economic loss was as large as millions and even billions [5].

The case of phenylpropanolamine strongly suggests the need of monitoring the pre-existing drugs available on the market. A drug's long history on the market does 
not mean its safety and efficacy meet the standards. It shows that FDA should not only focus on the identity of the new drugs proposed, but also frequently examine the performance of existing drugs to avoid any limbo.

\subsubsection{Deadly combination of Mibefradil and 25 other drugs}

Although only administered to around 200,000 patients, mibefradil was related to 123 fatalities in just one year on the market. As it passes the clinical trials, Mibefradil was approved by the FDA and considered generally safe when taken alone, but when coupled with any of 25 different medications, it became potentially lethal. Only ten months after FDA's approval, it had been withdrawn from the market owing to severe health risks discovered in post-release research [5].

The case of mibefradil warns the FDA that medicines should not be rushed to the market. If mibefradil had been held for a longer time, its adverse effects would be very likely to be discovered. Then, these deaths of innocent people would not occur. On the other hand, although the regulations and the process of drug approval are very complicated with high standards, there are still some defects. In this case, the deadly combination of mibefradil and 25 other drugs could be hard to discover during the clinical trials as the participants might not have taken any of these drugs to provide evidence of this situation. In other words, drug companies and the FDA should examine the relationship between the newly designed drug and other drugs available on the market to avoid conditions like mibefradil.

\section{PHARMACEUTICAL INDUSTRY MANAGEMENT DEPARTMENT}

\subsection{The State Food and Drug Administration}

The main functions of the SFDA Food and Drug Administration are as follows:

(1) Formulate policies, plans and supervise the implementation of the supervision and administration of drugs and medical devices, and participate in the drafting of relevant laws and regulations and draft departmental rules. (2) Responsible for the administrative supervision and technical supervision of drugs and medical devices, formulating quality management norms and implementation of the development, production, circulation and use of drugs and medical devices. (3) Responsible for the registration and supervision of drugs and medical devices, formulate national drug and medical device standards and supervise implementation, organize adverse drug reactions and adverse events, reevaluation and elimination of drugs and medical devices, participate in the formulation of national essential drug catalog, cooperate with relevant departments to implement the national essential drug system, and organize and implement the classified management system of prescription and non-prescription drugs. (4) Responsible for formulating and organizing implementation of TCM and ethnic drugs, formulating quality standards of Chinese medicine and ethnic drugs, organizing and formulation of production quality management standards of Chinese medicinal materials, processing norms and implementation of Chinese medicine decoction pieces and tablets, and organizing and implementation of the protection system of Chinese medicine varieties. (5) Supervise and manage the quality and safety of drugs and medical devices, supervise and manage radioactive drugs, narcotic drugs, toxic drugs and psychotropic drugs, and release the quality and safety information of drugs and medical devices [11].

\subsection{The National Health and Family Planning Commission}

This institution is responsible for formulating the development strategy and long-term planning of the pharmaceutical industry, conducting macro-control of the economic operation of the pharmaceutical industry, statistics and information of the pharmaceutical industry, pharmaceutical reserve and emergency dispatch; provincial and municipal health departments are responsible for drug bidding and procurement in their respective regions. Organize the formulation of national drug policies and the national essential drug system, organize the formulation of the national catalogue of essential drugs, formulate national management systems for the procurement, distribution and use of essential drugs, and participate in the formulation of the pharmacopoeia.

\subsection{The National Development and Reform Commission}

The National Development and Reform Commission is responsible for formulating drug price policies, supervising the implementation of price policies, and regulating the total level of drug prices.

\subsection{The National Ministry of Human Resources and Social Security}

The Ministry of Human Resources and Social Security is responsible for formulating the rules and policies for medical insurance and compiling the Drug Catalogue of National Basic Medical Insurance, Work-related Injury Insurance and Maternity Insurance [8]. 


\section{THE SIGNIFICANCE OF THE PHARMACEUTICAL INDUSTRY REGULATION}

\subsection{General overview}

Due to the particularity of the pharmaceutical industry, the state has formulated an extremely strict access and supervision system for each link of the industry from research and production to pricing and sales. In China has introduced relevant laws for the drug production, business license system, management system, national drug standard system, drug registration management system, drug price management system, prescription drugs and over-the-counter drug classification management system, drug intellectual property protection system and Chinese traditional Chinese medicine and so on. From then on, it can be seen that the government has devoted great efforts to drug supervision, which is not difficult to understand that due to the rapid development of society, people's living standards and accelerated pace of life, these things are not promoting economic and technological development and also putting pressure on people's health. Timely treatment and the appropriate medicine is the fastest way to solve people's pain and trouble, so drugs are very critical to treatment. Drugs are important, not only is itself but also the quality of the drug and the price it is sold. The quality of drugs is directly linked to people's health and life safety, and the price of drugs determines whether people can really do the right medicine, and take the most effective and most suitable medicine.

\subsection{Reflection originating from the harm}

The National Food and Drug Administration (SFDA) has also formulated some management norms for the quality management of drug production. However, in recent years, drug safety accidents and false drugs still occur from time to time. Most of the problems lie in the irregular behavior of agents, mainly manifested as corruption and bribery and the production of around enterprises. In order to maximize the benefit of a pursuit of sales, agents use more money to bribe doctors. With the escalation of this unhealthy atmosphere, the kickbacks of doctors increased, the interests of agents were reduced, manufacturers had to "make up" by reducing production costs, and eventually the quality of the drug became a victim after weighing the pros and cons. Agents seek their own interests, such as inducing pharmaceutical enterprises to change the drug production process or imitating well-known brand drugs are a common means in the market. The contradiction between the demand for drug safety and the development of the drug industry is inevitable. While the victims of the people, the social attention of the medical industry is also increased as unprecedented, and the drug supervision departments and drug manufacturers are facing unprecedented pressure. The people's health is to be guaranteed with the corresponding quality of high-quality drugs, and the product quality is determined by the production process of pharmaceutical enterprises. Therefore, the first person responsible for product quality must be a pharmaceutical company. To ensure the quality of drugs, drug manufacturers must strengthen their own management [10].

\section{STRATEGIES AND SUGGESTIONS ON IMPROVING THE EFFECTIVE REGULATORY CONTROL OF THE PHARMACEUTICAL INDUSTRY}

\subsection{Start with enterprises to improve drug production}

In today's society, the market competition is particularly fierce, if only using low prices to attract consumers is very low efficiency and cannot stand out from many enterprises. Compared with the profit in price, the pursuit of quality is obviously a better marketing means, which is also a kind of benign competition more conducive to the long-term development of the drug market. If companies are committed to finding the highest quality in the budget to gain customer favor, then it means that all the drugs produced are guaranteed, and people's safety and health are guaranteed. Practice has proved that many large domestic enterprises are from paying close attention to product quality only gradually. For example, in Qingdao Haier, Zhang Ruimin has cut off the production line of low and substandard drugs and paid close attention to quality inspection. Therefore, it makes Haier become a well-known international brand today. For example, many other practices have proved that the success of many large domestic enterprises cannot be separated from the reputation guarantee brought by good quality. As for improving the level of quality inspection of the company, multi-level quality analysis seminars can be used to discuss the problems of observing the assembly line, timely summarize and improve and clear employee rules and regulations to avoid corners or other unnecessary behaviors that affect the quality of drugs, and pursue higher efficiency from both aspects of people and material $[12,13]$.

\subsection{Correct the atmosphere of medical professionals}

People's greed is infinite, but when in a conflict of interest, people will also quickly make their own more beneficial choices. So starting from this point, the government should strengthen the punishment for corruption and bribery, so that they can be truly deterred 
by the punishment intensity and stop the continuation of the bad atmosphere. Of course, in another aspect, the investigation of investigation should also be strengthened, after all, if there is an empty law but the criminals, then no matter how strict the law can only be a decoration [14].

\subsection{Talent construction improves drugs}

The production and operation activities of pharmaceutical production enterprises are all completed by personnel. Therefore, personnel is the primary condition for drug production, is the most active and active factor in production factors. As a pharmaceutical production enterprise should be equipped with the corresponding quality and quantity of management personnel, technical personnel and production personnel, should establish the requirements of various specifications, according to the improvement of drug production quality management organization, effectively allocate enterprise resources, ensure the timely communication within the organization, and clearly allocate power and responsibility, so that the overall organization in an orderly direction of strategic success. In addition to introducing new talents, the company's internal training is also a very convenient and long-term means to improve the level of the company's staff. Therefore, pharmaceutical enterprises should regularly launch regular discussion and training, such as new and old employee exchange and experience sharing, professional knowledge transfer of technical personnel, as well as regular assessment to increase employee sense of urgency. Appropriate pressure is a good medicine for progress. The group building activities are also conducive to the communication and communication between employees and employees, and contribute to the creation of better teamwork. As for the assessment system of survival, it can help the company choose the best and find more training talents.

\section{CONCLUSION}

As discussed in this paper, the framework of the approval system designed by the FDA is well-thought and carried out to avoid many cases of drug failure and deaths. With no doubt, legislation is one of the most essential components of public health. It aims to protect the public's health by ensuring the quality, safety, and efficacy of medications and treatment. Without the intervention of regulatory practices or drug administrative authorities like the FDA, cases of fatal drugs or drugs causing other diseases would be much more prevalent across the world. It is very important that every medicine is tested and approved before it enters the market. Patients purchase drugs, hoping to cure their diseases, but if medicines on the market cannot be trusted, the drugs might not help them. Instead, the drug would kill them. Meanwhile, the public's trust in drugs on the market would be diminished after several cases of failure, causing small diseases to be not treated with the correct medication and even trigger more unnecessary loss. In order words, the administration of drugs is essential. As technology advances, the FDA's monitoring and regulation will continue to be critical in ensuring that patients have access to safe and effective therapies in the future.

\section{AUTHORS' CONTRIBUTIONS}

This paper is completed by Qianyin Liu and Haozhe Zhu together.

\section{ACKNOWLEDGMENTS}

At the end of the essay, I will express my sincere appreciate to all those who have helped me. First thank professors. Axel from Cambridge and other teachers who guide relevant revisions to our paper.Finally, thanks to raising our family, without whom we cannot have such good learning conditions to conduct relevant academic research.

\section{REFERENCES}

[1] Centennial of FDA, Aug 4, 2021. https://web.archive.org/web/20160524231757/https ://www.fda.gov/AboutFDA/

WhatWeDo/History/CentennialofFDA/default.htm.

[2] Stevens, C. W. Snake Eyes: Gambling with the Adverse Effects Of Ambien®. The Drug Expert 2020, 129-137.

[3] Fang, W. H.; Vangsness, C. T. Governmental Regulations and Increasing Food and Drug ADMINISTRATION Oversight of Regenerative MEDICINE Products: What's New in 2020? Arthroscopy: The Journal of Arthroscopic \& Related Surgery, 2020, 36 (10), 2765-2770.

[4] Osakwe, O. Pharmaceutical Regulation: The Role of Government in the Business of Drug Discovery. Social Aspects of Drug Discovery, Development and Commercialization, 2016, 3-27.

[5] McIntyre, D. A. The ten worst Drug recalls in the history of The FDA, 2010. https://247wallst.com/investing/2010/12/10/the-ten -worst-drug-recalls-in-the-history-of-the-fda/\#ixz2 ZfkHjNzQ (accessed Aug 4, 2021).

[6] Editors of Thalidomide Victims Association of Canada. What is thalidomide? https://thalidomide.ca/en/what-is-thalidomide/ (accessed Aug 4, 2021).

[7] Junod, S. W. FDA and Clinical Drug Trials: A Short History, 2008. 
[8] Zheng Xiaoyu, implement the New Drug Law to further strengthen drug supervision and management [C] 2001 Academic Annual Meeting of Chinese Pharmaceutical Association, January 11, 2001.

[9] Zhang Weixiu, briefly on the quality management of drug production.Shanghai Qingan Pharmaceutical Group Suzhou Pharmaceutical Co., Ltd., Suzhou, Anhui 234000, Ltd.

[10] Wang Yan, Chen Yongmei, Problems and Countermeasures in the Implementation of National Drug Quality Standards [J]. Anhui Pharmaceutical, 2007, 11 (4): 372.

[11] China Pharmaceutical Industry Corporation, Drug Production Quality Management Specification Practice Guidelines [S] Beijing: Chemical Industry Press, 2001:9.

[12] Zhu Shibin, Drug Production Quality Management Project [M] Beijing: Chemical Industry Press, 2001:31.

[13] Bai Huiliang, the necessity to strengthen drug supervision and management under the new situation [J] Division of Safety 100810, 2002 (Volume 5), Issue 7.

[14] Zhao Jianmei, the Necessity for Strengthening Drug Supervision and Management [J] Road to Health, 2014 (000), 011. 